\title{
Low Complexity Controllers for Vibrations Damping in Drilling Systems
}

\author{
Islam Boussaada and Hugues Mounier and Silviu-Iulian Niculescu
}

\begin{abstract}
In oilwell drillstring systems, vibrations represent an important source of economic losses; drill bit wear, pipes disconnection, borehole disruption and prolonged drilling time, are only a few examples of consequences associated with drilling vibrations. In this paper a control-oriented model of torsional vibrations occurring in rotary drilling process is proposed. More precisely, a wave equations with weak damping term is considered. An appropriate stabilizing controller with a reduced number of parameters is proposed for damping such torsional vibrations. Such a controller allows to further explore the effect of multiple roots with maximal admissible multiplicity for linear neutral system with a single delay. An illustrative example completes the presentation.
\end{abstract}

\section{INTRODUCTION}

It is commonly accepted that most processes include aftereffect phenomena in their dynamics and there exists an abundant literature devoted to delay models in economics, physiology, population dynamics, biology, chemistry, viscoelasticity, physics, information technologies, mechanics, engineering sciences, etc, see for instance [26] and the references therein.

It is well known that self-excited drillstring vibrations are the main cause of loss of performance in the perforation process for oil and gas; they provoke premature wear and tear of drilling equipment resulting in fatigue and induced failures such as pipe wash-out and twist-off [30].

Roughly speaking, self-excited vibrations can be explained by the instability of an equilibrium in the dynamic system description (see e.g. [28]). An unstable equilibrium point implies that a small initial system perturbation will grow over time until it manifests itself as the observable phenomena of stick-slip, in the case of torsional vibrations, or bitbouncing, in the case of axial vibrations [1], [6], [8], [33]. The bit-bounce phenomenon, caused by axial vibrations, is characterized by a repetitive loss of contact of the bit with the rock formation. The stick-slip effect, provoked by torsional vibrations, can be recognized by the successive occurrence of two phases: stick (the bit stops rotating) and slip (the bit is released from the stick phase with angular velocity higher

\footnotetext{
Islam Boussaada is with IPSA \& Inria Saclay, DISCO Team \& Laboratoire des Signaux et Systèmes, Université Paris Saclay, CNRS-CentraleSupélec-Université Paris Sud, 3 rue Joliot-Curie, 91192 Gif-sur-Yvette cedex, France Islam. Boussaadall2s. centralesupelec.fr

Hugues Mounier is with Laboratoire des Signaux et Systèmes, Université Paris Saclay, Université Paris Sud-CNRS-CentraleSupélec, 3 rue Joliot-Curie, 91192 Gif-sur-Yvette cedex, France, Hugues. Mouierel2s.centralesupelec.fr

Silviu-Iulian Niculescu is with Laboratoire des Signaux et Systèmes, Université Paris Saclay, CNRS-CentraleSupélec-Université Paris Sud, 3 rue Joliot-Curie, 91192 Gif-sur-Yvette cedex, France, Silviu.Niculescull2s.centralesupelec.fr

Manuscript received August 1st, 2018
}

than the nominal one) [27]. It has been reported that the stick-slip phenomenon occurs for about fifty percent of the drilling operation [25].

The harmful consequences of drillstring vibrations have motivated extensive research to analyze them and to try to mitigate their influence during drilling operations. In order to reduce the costs of failures, extensive research effort has been conducted in the last five decades to suppress drillstring vibrations and several methodologies have been proposed, both from practical and theoretical viewpoints, see the references cited in the survey [29] and in [24], [35].

In general, classical controllers are not necessarily able to stabilize delay systems. A common technique to avoid the complexity of delay systems consists in approximating them by finite-dimensional representations; however, neglecting aftereffects is not an appropriate solution: in the best case scenario it leads to the same degree of complexity in the control design, in worst situations it is potentially harmful for the system's stability. All these remarks motivated us first to provide a realistic control-oriented and relatively simple time-delay model for representing the dynamical behavior of the drilling vibrations. Next, we exploit the structure of the model and propose a controller with a reduced number of tuning gains (or parameters). Finally the controllers gains are chosen such that the spectrum of the closed-loop system has a a particular structure. Including multiple roots on the real axis surprisingly, such a spectral distribution leads to closed-loop stabilizing of the overall system. Such an idea was already exploited in the case of retarded systems, but not for neutral systems and it represents a novelty.

It is worth mentioning that the rightmost root for quasipolynomial function corresponding to stable retarded time-delay systems (also in the neutral case under some assumptions) is actually the exponential decay rate of its time-domain solution, see for instance [16] for an estimate of the decay rate for stable linear delay systems. To the best of the authors knowledge, the first time an analytical proof of the dominancy of a spectral value for the scalar equation with a single delay was presented in [17] and was further exploited by the authors. More precisely, the dominancy property is further discussed for scalar delay equations in [12] for second-order systems controlled by a delayed proportional is proposed in [18], [19]. Next, its applicability in damping active vibrations for a piezo-actuated beam is proved and in [20], [21] the regulation of the Mach number in a wind tunnel is considered.

This note provides a new exploration of multiple roots properties for time-delay systems of neutral type carried out from wave equations with a weak damping term correspond- 
ing to a viscous damping.

The paper is organized as follow. In Section II, a PDE model describing the torsional vibration in oilwell rotary drilling system is provided. Section III is devoted to the control design for damping such vibrations. An applicative illustrative example completes the paper. The paper ends by Some concluding remarks end the paper.

\section{PDE Modelling of the DRILling VibRations}

\section{A. A Coupled Axial/Torsional Vibrations Model}

It is well known that torsional vibrations contribute to the excitation of axial oscillations. In fact, these self-excited oscillations are intimately coupled together and may occur simultaneously. Coupled axial-torsional excitations of a drillstring of length $L$, described by the rotary angle $\Phi(s, t)$ and the longitudinal position $U(s, t)$ may be modeled by two wave equations [4]:

$$
\begin{aligned}
\partial_{z}^{2} \Phi(z, t) & =\tilde{c}^{2} \partial_{t}^{2} \Phi(z, t), \\
\partial_{z}^{2} U(z, t) & =c^{2} \partial_{t}^{2} U(z, t),
\end{aligned}
$$

with boundary conditions

$$
\begin{aligned}
G J \partial_{z} \Phi(0, t) & =\beta \partial_{t} \Phi(0, t)-u_{T}(t) \\
G J \partial_{z} \Phi(L, t) & =-I_{B} \partial_{t}^{2} \Phi(L, t)-T\left(\partial_{t} \Phi(L, t)\right),
\end{aligned}
$$

and

$$
\begin{aligned}
E \Gamma \partial_{z} U(0, t) & =\alpha \partial_{t} U(0, t)-u_{H}(t) \\
E \Gamma \partial_{z} U(L, t) & =-M_{B} \partial_{t}^{2} U(L, t)-T\left(\partial_{t} \Phi(L, t)\right) .
\end{aligned}
$$

The spatial variable " $z$ " is chosen such that $z=0$ denotes the top of the drillstring and $z=L$ its bottom. The propagation speeds of the axial and torsional waves $v_{U}, v_{\Phi}$, defined as: $v_{U}=c^{-1}$ and $v_{\Phi}=\tilde{c}^{-1}$ can be computed from the material parameters, namely the Young modulus $E$, the shear modulus $G$ and the density $\rho_{a}$, by means of

$$
c=\sqrt{\frac{\rho_{a}}{E}} \text { and } \tilde{c}=\sqrt{\frac{\rho_{a}}{G}} .
$$

In the boundary condition (3a), $u_{H}$ is the brake motor control (upward hook force) and $\alpha \partial U_{t}(0, t)$ represents a friction force of viscous type (where $\alpha$ is the viscous friction coefficient). In (2a), $u_{T}$ represents the torque produced by the rotary table motor and $\beta \partial_{t} \Phi(0, t)-u_{T}(t)$ designates the difference between the motor speed and rotational speed of the first pipe. It is assumed that the drilling system can be controlled by the boundary force $u_{H}$ and the boundary torque $u_{T}$.

The model contains some geometrical parameters of the drill string, that are assumed to be spatially and timely constant. These comprise the drillstring's cross-section $\Gamma$ and its second moment of area $J$, as well as the mass $M_{B}$ and the inertia moment ${ }^{1}$ of the drill bit $I_{B}$. The function $T$ considered in the bottom boundary conditions accounts for

\footnotetext{
${ }^{1}$ The inertia moment is such that $I_{B}=M_{B} r^{2}$, where $r$ is taken as the averaged radius of drillpipe.
}

the frictional torque resulting from the interaction between the drill bit and the rock.

It is important to point out that the boundary conditions ( $2 b)$ and ( $3 b$ ) corresponding to the bottom of the rod, involve a frictional torque arising from the bit-rock interaction. The modeling of the torque on the bit constitutes a crucial aspect of the system description since it allows to reproduce the vibrational phenomena. The reader can find the numerical values of the model's parameters in Appendix A.

\section{B. Torsional Vibrations: A Neutral-type Time-delay Model}

The wave equation model provides a realistic description of the distributed system variables; however, in some circumstances, it is convenient to deal with a relatively simpler model involving just the primary interest variables. This section presents a direct procedure to derive, from the wave equations, some equivalent input-output models described by neutral-type time-delay equations relating the variables at both ends of the drilling rod.

Integration along characteristics of the hyperbolic PDE allows the association of a certain system of functional differential equations to the mixed problem. More precisely, a one-to-one correspondence may be established and proved between the solutions of the mixed problem for hyperbolic PDE and the initial value problem for the associated system of functional equations [31]. By reducing a boundary value problem to a neutral-type time-delay equation we are able to exploit techniques from delay systems theory to gain insight into the complexity involved in the analysis and simulation of the corresponding PDE models. The PDE model we consider accompanied with boundary conditions is given by:

$$
\begin{aligned}
\partial_{z}^{2} \Phi(z, t) & -\gamma \partial_{t} \Phi(z, t)-c^{2} \partial_{t}^{2} \Phi(z, t)=0 \\
G J \partial_{z} \Phi(0, t) & =\beta \partial_{t} \Phi(0, t)-u_{T}(t) \\
G J \partial_{z} \Phi(L, t) & =-I_{B} \partial_{t}^{2} \Phi(L, t)-T\left(\partial_{t} \Phi(L, t)\right)
\end{aligned}
$$

which can be reducible to an I/O system. Namely, as described in Appendix C, (5) reduces to a neutral system of order 3 with a single delay $\tau=c L$ that can be written as:

$$
\left\{\begin{array}{l}
\left(-\frac{\beta I_{B}}{\alpha_{L} 2 G J c}-\frac{I_{B}}{2 \alpha_{L}}\right) \dddot{\Phi}_{L}(t)+\left(\frac{\beta I_{B} \alpha_{L}}{2 G J c}-\frac{I_{B} \alpha_{L}}{2}\right) \dddot{\Phi}_{L}(t-2 \tau) \\
-\left(\frac{\beta}{2 \alpha_{L}}+\frac{G J c}{2 \alpha_{L}}\right) \ddot{\Phi}_{L}(t)+\left(\frac{G J c}{2} \alpha_{L}-\frac{\beta \alpha_{L}}{2}\right) \ddot{\Phi}_{L}(t-2 \tau) \\
-\left(2 \frac{G J c}{2 \alpha_{L}} \zeta+\frac{\beta}{2 \alpha_{L}}\right) \dot{\Phi}_{L}(t) \\
+\left(2 \frac{G J c \zeta \alpha_{L}}{2}-\frac{\beta \zeta \alpha_{L}}{2}\right) \dot{\Phi}_{L}(t-2 \tau) \\
+\frac{\zeta^{2} G J c}{2 \alpha_{L}} \Phi_{L}(t)+\frac{\zeta^{2} G J c}{2 \alpha_{L}} \Phi_{L}(t-2 \tau) \\
=\frac{1}{2}\left(\alpha_{L} \ddot{\Phi}_{L}(t-2 \tau)+\alpha_{L}^{-1} \ddot{\Phi}_{L}(t)\right) \times \\
T^{\prime}\left(\alpha_{L} \dot{\Phi}_{L}(t-2 \tau)+\alpha_{L}^{-1} \dot{\Phi}_{L}(t)\right) \\
-\left(\alpha_{L} \ddot{\Phi}_{L}(t-2 \tau)-\alpha_{L}^{-1} \ddot{\Phi}_{L}(t)\right) \times \\
T^{\prime}\left(\alpha_{L} \dot{\Phi}_{L}(t-2 \tau)-\alpha_{L}^{-1} \dot{\Phi}_{L}(t)\right) \\
-\dot{u}_{T}(t-\tau)-\zeta u_{T}(t-\tau) .
\end{array}\right.
$$


It is important to point out that the delay $\tau$ represents the time that the torsional wave takes to travel from one to the other extremity of the drillstring, see for instance Appendix $\mathrm{B} \& \mathrm{C}$.

\section{LOW COMPLEXITY CONTROLLER DESIGN}

The multiplicity induced-dominancy (MID) property was underlined in [12], [18] for retarded time-delay systems and were exploited in several applications [19], [20], [21]. The controller we propose for suppressing torsional vibrations is of the form $u_{T}(t)=\kappa_{1} \Phi_{L}(t-\tau)$ where the delay is the same as the one intrinsic to the model. This provides a new extension in the applicability of MID property for time-delay systems of neutral type carried out from wave equations with a weak damping term corresponding to a viscous damping. The design approach we consider is a frequency-domain method that consists in intentionally forcing the characteristic equation to have multiple negative spectral values. Under appropriate conditions, such a multiple root may be the dominant one among the spectrum, guaranteeing thus the stability of the closed-loop system.

Let us first, recall some important facts. In frequency domain, we deal with linear functional differential equations only. Thus, we consider the linearization of (6), which gives:

$$
\left\{\begin{array}{l}
\left(-\frac{\beta I_{B}}{\alpha_{L} 2 G J c}-\frac{I_{B}}{2 \alpha_{L}}\right) \dddot{\Phi}_{L}(t)+\left(\frac{\beta I_{B} \alpha_{L}}{2 G J c}-\frac{I_{B} \alpha_{L}}{2}\right) \dddot{\Phi}_{L}(t-2 \tau) \\
-\left(\frac{\beta}{2 \alpha_{L}}+\frac{G J c}{2 \alpha_{L}}\right) \ddot{\Phi}_{L}(t)+\left(\frac{G J c}{2} \alpha_{L}-\frac{\beta \alpha_{L}}{2}\right) \ddot{\Phi}_{L}(t-2 \tau) \\
-\left(2 \frac{G J c}{2 \alpha_{L}} \zeta+\frac{\beta}{2 \alpha_{L}}\right) \dot{\Phi}_{L}(t)+\left(2 \frac{G J c \zeta \alpha_{L}}{2}-\frac{\beta \zeta \alpha_{L}}{2}\right) \dot{\Phi}_{L}(t-2 \tau) \\
+\frac{\zeta^{2} G J c}{2 \alpha_{L}} \Phi_{L}(t)+\frac{\zeta^{2} G J c}{2 \alpha_{L}} \Phi_{L}(t-2 \tau) \\
=\frac{1}{\bar{k}}\left(\alpha_{L} \ddot{\Phi}_{L}(t-2 \tau)+\alpha_{L}^{-1} \ddot{\Phi}_{L}(t)\right) \\
-\frac{2}{\bar{k}}\left(\alpha_{L} \ddot{\Phi}_{L}(t-2 \tau)-\alpha_{L}^{-1} \ddot{\Phi}_{L}(t)\right) \\
-\dot{u}_{T}(t-\tau)-\zeta u_{T}(t-\tau),
\end{array}\right.
$$

since the friction force at the bottom denoted by $T$ is modeled in [22] by the following odd function: $T(x)=$ $\frac{2 \bar{k} x}{k^{2}+x^{2}}$, for which corresponds the local approximation:

$$
T(x)=\frac{2}{\bar{k}} x+O\left(x^{3}\right),
$$

See also [23] for further discussion on friction models in rotary drilling systems.

Consider now a controller $u_{T}(t)=\kappa_{1} \Phi_{L}(t-\tau)$, then $\dot{u}_{T}(t)=\kappa_{1} \dot{\Phi}_{L}(t-\tau)$ and system (8) writes in closed-loop as:

$$
\left\{\begin{array}{l}
a_{3} \dddot{\Phi}_{L}(t)+b_{3} \dddot{\Phi}_{L}(t-2 \tau) \\
-a_{2} \ddot{\Phi}_{L}(t) \\
+b_{2} \ddot{\Phi}_{L}(t-2 \tau) \\
-a_{1} \dot{\Phi}_{L}(t)+b_{1} \dot{\Phi}_{L}(t-2 \tau) \\
+a_{0} \Phi_{L}(t)+b_{0} \Phi_{L}(t-2 \tau)=0
\end{array}\right.
$$

where:

$$
\left\{\begin{aligned}
a_{3} & =-\frac{\beta I_{B}}{\alpha_{L} 2 G J c}-\frac{I_{B}}{2 \alpha_{L}}, b_{3}=\frac{\beta I_{B} \alpha_{L}}{2 G J c}-\frac{I_{B} \alpha_{L}}{2}, \\
a_{2} & =-\frac{\beta}{2 \alpha_{L}}-\frac{G J c}{2 \alpha_{L}}-\frac{3}{\bar{k} \alpha_{L}}, b_{2}=\frac{G J c}{2} \alpha_{L}-\frac{\beta \alpha_{L}}{2}+\frac{\alpha_{L}}{\bar{k}}, \\
a_{1} & =-\frac{G J c}{\alpha_{L}} \zeta-\frac{\beta}{2 \alpha_{L}}, b_{1}=\kappa_{1}+G J c \zeta \alpha_{L}-\frac{\beta \zeta \alpha_{L}}{2}, \\
a_{0} & =\frac{\zeta^{2} G J c}{2 \alpha_{L}}, b_{0}=\kappa_{1} \zeta+\frac{\zeta^{2} G J c}{2 \alpha_{L}} \quad \text { where } \\
c & =\sqrt{\frac{\rho_{a}}{G}}, \zeta=\frac{\gamma}{2 c^{2}}, \alpha_{L}=e^{-c L \zeta}, \tilde{\tau}=2 \tau=2 c L,
\end{aligned}\right.
$$

and the numerical values of the remaining parameters are given in Table I in Appendix.

Such a system is of neutral type and it can be rewritten as a quasipolynomial including one delay using the Laplace transform.

$$
\left\{\begin{array}{l}
L(s)=P(s)+Q(s) e^{-s \tilde{\tau}} \\
P(s)=\sum_{i=0}^{3} a_{k} s^{k} \\
Q(s)=\sum_{i=0}^{3} b_{k} s^{k}
\end{array}\right.
$$

The main result can be resumed as follows.

Proposition 1. The linearized system (8) subject to the controller $u_{T}(t)=\kappa_{1} \Phi_{L}(t-\tau)$ has the following properties in closed-loop :

- The maximal multiplicity of any root of the quasipolynomial $L$ given in (10) with arbitrary coefficients $\left(a_{k}, b_{k}\right)_{0 \leq k \leq 3}$ is bounded by 7 .

- If $b_{k}<1 \forall 0 \leq k \leq 3$ and such a maximal multiplicity is reached, then the corresponding spectral value is real, negative and dominant.

Sketch of the proof: Exploring the multiplicity 7 suggests to consider the quasipolynomial $L$ as well as its first six derivatives. More precisely, setting $L(s)=0$ allows to eliminate the exponential term and writing it as a rational function in $s$. Taking this into account allows to say that the vanishing of the first six derivatives of $L$ represents an algebraic ${ }^{2}$ system of six equations. Solving them, gives a single solution in one free parameter. Such a solution is then substituted in the first elimination equation which gives the value of the remaining parameter and then the complete solution (without free parameters). Substituting such a final solution in the seventh derivative of $L$ shows that $L^{(7)}\left(s_{0}\right) \neq 0$. To show the dominancy of $s_{0}$ one uses the principle argument. The condition on $b_{k}<1$ guarantees the asymptotic axis of spectrum chain is either in the open left half-plane or is the imaginary axis, see [7].

\section{ILLUSTRATIVE EXAMPLE}

Since the system's parameters are initially fixed, then the maximal multiplicity cannot be reached. We will apply the

${ }^{2}$ polynomials in $s$ parametrized by $a_{k}$ and $b_{k}$ 


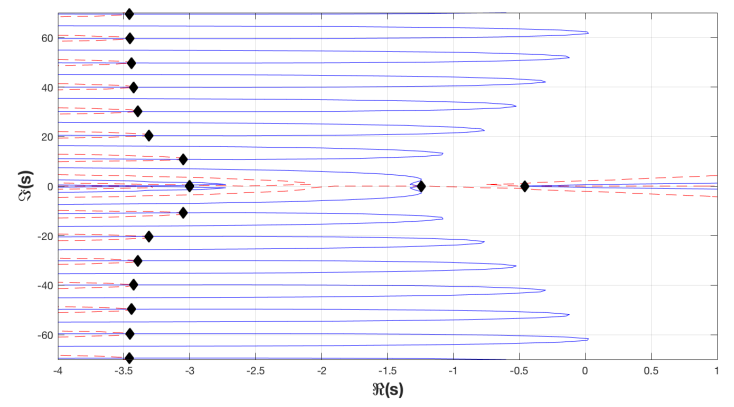

Fig. 1. The spectral values' distribution for equation (8) is illustrated using QPmR toolbox from [15]. The four rightmost roots correspond to non oscillating solution are emphasized (a double root at $s=-3$, a simple root near $s=-0.5$ and a simple root near $s=-1.3$.

idea outlined in the previous section

Proposition 2. If the system parameters are chosen in Table $I$ and

$$
\begin{aligned}
b_{0}= & 0.850229 a_{1}-1.212251 a_{2}-0.378553 a_{3} \\
& -0.432124 a_{0}-54 b_{3}+9 b_{2}, \\
b_{1}= & 6 b_{2}+0.134694 a_{1}+0.042061 a_{2}-1.464620 a_{3} \\
& -0.094469 a_{0}-27 b_{3} \text { and the gain } \\
\kappa_{1}= & -2854.883393,
\end{aligned}
$$

then $s_{0}=-3$ is a double root for (10). Furthermore, in such a case the trivial solution is asymptotically stable.

Remark. It is important to observe that, even if the multiple root is not dominant, the closed loop-system is asymptotically stable. Figure 1 illustrates the spectral distribution of the characteristic function (10) with the parameters defined in Table 1 and Proposition 2.

\section{CONCLUDING REMARKS}

This paper presents a PDE modeling of torsional vibration in rotary drilling system. Such a model is then reduced to a third-order neutral equation with a single delay. for which a reduced complexity delayed stabilizing controller is proposed for an appropriate pole-placement of the closed-loop system. The obtained controller acts for damping oscillation of the wave equation.

\section{ACKNOWLEDGEMENTS}

The authors would like to thank the anonymous referees for their constructive remarks on improving the presentation of the paper. This research was partially supported by the iCODE Institute, research project of the IDEX ParisSaclay, and by the Hadamard Mathematics LabEx (LMH) through the grant number ANR-11-LABX-0056-LMH in the "Programme des Investissements d'Avenir". IB \& SIN are partially financially supported by a grant from Hubert Curien (PHC) BRANCUSI 2017, project number 38390ZL and a grant from PHC BALATON 2018, project number 40502NM.

\section{APPENDIX A: NUMERICAL VALUES OF THE MODEL'S PARAMETERS}

See Table 1.

TABLE I

NUMERICAL VALUES OF SOME OF THE DRILLING SYSTEM PARAMETERS.

\begin{tabular}{ccc}
\hline \hline Symbol & Parameter & Numerical value \\
\hline$L$ & String length & $1000 \mathrm{~m}$ \\
$G$ & Shear modulus & $79.3 \times 10^{9} \mathrm{~N} \mathrm{~m}^{-2}$ \\
$\Gamma$ & Drillstring's cross-section & $35 \times 10^{-4} \mathrm{~m}^{2}$ \\
$J$ & Second moment of area & $1.19 \times 10^{-5} \mathrm{~m}^{4}$ \\
$I_{B}$ & Lumped inertia at the BHA & $89 \mathrm{Kg} \mathrm{m}^{2}$ \\
$\rho_{a}$ & Density & $8000 \mathrm{Kg} \mathrm{m}^{-3}$ \\
$\beta$ & Angular momentum & $2000 \mathrm{~N} \mathrm{~m} \mathrm{~s}$ \\
$\gamma$ & Damping constant & $6,0529634310^{-7} \frac{\mathrm{Nms}}{\mathrm{rad}}$ \\
$\bar{k}$ & Constant of the friction top angle & $2.610^{-3}$
\end{tabular}

\section{APPENDIX B: WAVE EQUATION WITH A WEAK DAMPING} TERM

A torsional model improving (1a) accompanied with the boundary conditions (2) is given by:

$$
\begin{aligned}
\partial_{z}^{2} \Phi(z, t) & -\gamma \partial_{t} \Phi(z, t)-c^{2} \partial_{t}^{2} \Phi(z, t)=0 \\
G J \partial_{z} \Phi(0, t) & =\beta \partial_{t} \Phi(0, t)-u_{T}(t) \\
G J \partial_{z} \Phi(L, t) & =-I_{B} \partial_{t}^{2} \Phi(L, t)-T\left(\partial_{t} \Phi(L, t)\right)
\end{aligned}
$$

The temporal Laplace transform of (11a) yields

$$
\left(\partial_{z}^{2}-\gamma s-s^{2} c^{2}\right) \hat{\Phi}(z, s)=0
$$

leading to the characteristic equation $\xi^{2}-\gamma s-s^{2} c^{2}=0$ or equivalently, $\xi=\sqrt{\gamma s+s^{2} c^{2}}$.

Since the damping coefficient is assumed to be sufficiently small $\gamma \ll 1$, then

$$
\xi= \pm c s \sqrt{1+\frac{\gamma}{c^{2}} \frac{1}{s}}= \pm \sqrt{1+\frac{\eta}{d}}
$$

where $\eta=\frac{\gamma}{c^{2}}$. This allows to:

$$
\xi= \pm\left(c s+\frac{c \eta}{2}\right)+o(\eta),
$$

Thus, $\xi= \pm(c s+c \zeta)$ with $\zeta=\frac{\eta}{2}=\frac{\gamma}{2 c^{2}}$ which corresponds to the following characteristic equation:

$$
\xi^{2}-c^{2}(s+\zeta)^{2}=0
$$

or equivalently: $\xi^{2}-c^{2} s^{2}-2 c^{2} \zeta s-\zeta^{2}=0$ itself corresponds to the following partial differential equation

$$
\partial_{z}^{2} \Phi(z, t)-2 c^{2} \zeta \partial_{t} \Phi(z, t)-c^{2} \partial_{t}^{2} \Phi(z, t)-\zeta^{2} \Phi(z, t)=0 .
$$

Taking $\xi= \pm c(s+\zeta)$, the general solution of (12) is given by

$$
\begin{aligned}
\hat{\Phi}(z, s) & =e^{c z(s+\zeta)} \hat{\mu}_{1}(s)+e^{-c z(s+\zeta)} \hat{\mu}_{2}(s) \\
& =e^{c z s} e^{c z \zeta} \hat{\mu}_{1}(s)+e^{-c z s} e^{-c z \zeta} \hat{\mu}_{2}(s)
\end{aligned}
$$

which is to be compared to the solution of (12) with $\zeta=0$ (the undamped wave equation) corresponding to the 
d'Alembert solution $e^{c z s} \hat{\mu}_{1}(s)+e^{-c z s} \hat{\mu}_{2}(s)$. In conclusion, in the case of a weak damping term, the solution of the undamped equation represents a relatively good approximation for the solutions of the wave equation with damping term, see for instance [2].

\section{APPENDIX C: CARRYING-OUT A FLAT OUTPUT FOR THE PDE MODEL}

The PDE model we consider accompanied with boundary conditions is given by:

$$
\begin{aligned}
\partial_{z}^{2} \Phi(z, t) & -\gamma \partial_{t} \Phi(z, t)-c^{2} \partial_{t}^{2} \Phi(z, t)=0 \\
G J \partial_{z} \Phi(0, t) & =\beta \partial_{t} \Phi(0, t)-u_{T}(t) \\
G J \partial_{z} \Phi(L, t) & =-I_{B} \partial_{t}^{2} \Phi(L, t)-T\left(\partial_{t} \Phi(L, t)\right)
\end{aligned}
$$

which can be reducible to : Knowing that the general solution of (14) can be expressed as

$$
\begin{aligned}
& \hat{\Phi}_{z}=\hat{C}_{z} \hat{\mu}_{1}+\hat{S}_{z} \hat{\mu}_{2}, \\
& \hat{\Phi}_{z}^{\prime}=c^{2}(s+\zeta)^{2} \hat{S}_{z} \hat{\mu}_{1}+\hat{C}_{z} \hat{\mu}_{2},
\end{aligned}
$$

where $\hat{\Phi}_{z}$ is nothing but the Laplace transform of $\Phi_{z}=$ $\Phi(z, t)$ and $\Phi_{z}^{\prime}$ is nothing but $\partial_{z} \Phi_{z}$ and

$$
\begin{aligned}
& \hat{C}_{z}=\cosh (c(z-L)(s+\zeta)), \\
& \hat{S}_{z}=\frac{\sinh (c(z-L)(s+\zeta))}{c(s+\zeta)},
\end{aligned}
$$

and $\hat{C}_{z}^{\prime}=c^{2}(s+\zeta)^{2} \hat{S}_{z}, \quad \hat{S}_{z}^{\prime}=\hat{C}_{z}$.

Finally, since $\hat{C}_{L}=1$ and $\hat{S}_{L}=0$, then, $\hat{\Phi}_{L}=\hat{\mu}_{1}$ and $\hat{\Phi}_{L}^{\prime}=\hat{\mu}_{2}$.

Let us now reconstruct a basis or a flat output of the model (5), see for instance [3], [32], [34]. The considered boundary conditions (2) rewrite

$$
\begin{aligned}
& G J \Phi_{0}^{\prime}=\beta \dot{\Phi}_{0}-u_{T}(t) \\
& G J \Phi_{L}^{\prime}=-I_{B} \ddot{\Phi}_{L}-T\left(\dot{\Phi}_{L}\right),
\end{aligned}
$$

which shows that $\Phi_{L}$ is a basis or flat output, see [5]. Indeed, one has

$$
\begin{aligned}
\mu_{1} & =\Phi_{L}, \\
\mu_{2} & =-\frac{I_{B}}{G J} \ddot{\Phi}_{L}-\frac{1}{G J} T\left(\dot{\Phi}_{L}\right)
\end{aligned}
$$

which allow us to express the variables at the top $\Phi_{0}, \dot{\Phi}_{0}, \Phi_{0}^{\prime}$ :

$$
\begin{aligned}
& \Phi_{0}=C_{0} \Phi_{L}-\frac{1}{G J} S_{0}\left(I_{B} \ddot{\Phi}_{L}+T\left(\dot{\Phi}_{L}\right)\right) \\
& \Phi_{0}^{\prime}=c^{2}\left(\frac{d}{d t}+\zeta\right)^{2} S_{0} \Phi_{L}-\frac{C_{0}}{G J}\left(I_{B} \ddot{\Phi}_{L}+T\left(\dot{\Phi}_{L}\right)\right) \\
& \dot{\Phi}_{0}=C_{0} \dot{\Phi}_{L}-\frac{1}{G J} \frac{d}{d t} S_{0}\left(I_{B} \ddot{\Phi}_{L}+T\left(\dot{\Phi}_{L}\right)\right)
\end{aligned}
$$

To establish the operators expressions, let us set $\alpha_{L}=e^{-c L \zeta}$ and $\hat{\delta}_{L}=e^{-c L s}$ with $\left(\delta_{L} w\right)=w(t-L c)$. Then

$$
\begin{aligned}
& \hat{C}_{0}=\cosh (c L(s+\zeta))=\frac{1}{2}\left(\alpha_{L}^{-1} \delta_{L}^{-1}+\alpha_{L} \delta_{L}\right) \\
& \hat{S}_{0}=-\frac{\sinh (c L(s+\zeta))}{c(s+\zeta)}=\frac{e^{c L \zeta} e^{-c L s}}{2 c} \frac{1-e^{-2 c L(s+\zeta)}}{s+\zeta} .
\end{aligned}
$$

Setting $\hat{\sigma}_{\beta, h}=\frac{1-e^{-h(s-\beta)}}{s-\beta}$ with $\left(\sigma_{\beta, h} w\right)(t)=$ $\int_{t-h}^{t} e^{\beta(t-\tau)} w(\tau) d \tau$, we have:

$$
\begin{aligned}
S_{0} & =-\frac{\delta_{L}^{-1} \sigma_{-\zeta, 2 c L}}{2 c \alpha_{L}}, \\
\left(\frac{d}{d t}+\zeta\right) S_{0} & =\frac{\alpha_{L} \delta_{L}-\alpha_{L}^{-1} \delta_{L}^{-1}}{2 c} \\
\frac{d}{d t} S_{0} & =\frac{\alpha_{L} \delta_{L}-\alpha_{L}^{-1} \delta_{L}^{-1}}{2 c}-\zeta S_{0} .
\end{aligned}
$$

We are now able to establish a top to bottom relation which allows to write our PDE system as an I/O system. Indeed, the input -output system, with input $u_{T}$ and output $\Phi_{L}$ which is a flat output. As a matter of fact, using the boundary condition (15) and substituting the expression of $\dot{\Phi}_{0}$ and $\Phi_{0}^{\prime}$ from (17) one obtains:

$$
\begin{aligned}
& \frac{G J c}{2}\left(\frac{d}{d t}+\zeta\right)\left(\alpha_{L} \delta_{L}-\alpha_{L}^{-1} \delta_{L}^{-1}\right) \Phi_{L} \\
& -\frac{1}{2}\left(\alpha_{L} \delta_{L}+\alpha_{L}^{-1} \delta_{L}^{-1}\right)\left(I_{B} \ddot{\Phi}_{L}+T\left(\dot{\Phi}_{L}\right)\right) \\
= & \frac{\beta}{2}\left(\alpha_{L} \delta_{L}+\alpha_{L}^{-1} \delta_{L}^{-1}\right) \dot{\Phi}_{L} \\
& -\frac{\beta}{G J}\left(\frac{1}{2 c}\left(\alpha_{L} \delta_{L}-\alpha_{L}^{-1} \delta_{L}^{-1}\right)-\zeta S_{0}\right)\left(I_{B} \ddot{\Phi}_{L}+T\left(\dot{\Phi}_{L}\right)\right) \\
& -u_{T} .
\end{aligned}
$$

All these steps allow to alleviate the PDE system into a delay system. Let us first set :

$$
\begin{aligned}
& \mathcal{D}=\frac{d}{d t}+\zeta, \\
& \Delta=\alpha_{L} \delta_{L}+\alpha_{L}^{-1} \delta_{L}^{-1}, \\
& \tilde{\Delta}=\alpha_{L} \delta_{L}-\alpha_{L}^{-1} \delta_{L}^{-1} .
\end{aligned}
$$

Then equation (19) rewrites

$$
\begin{aligned}
& \frac{G J c}{2} \mathcal{D} \tilde{\Delta} \Phi_{L}-\frac{1}{2} \Delta\left(I_{B} \ddot{\Phi}_{L}+T\left(\dot{\Phi}_{L}\right)\right) \\
& =\frac{\beta}{2} \Delta \dot{\Phi}_{L}-\frac{\beta}{G J}\left(\frac{\tilde{\Delta}}{2 c}-\zeta S_{0}\right)\left(I_{B} \ddot{\Phi}_{L}+T\left(\dot{\Phi}_{L}\right)\right)-u_{T}
\end{aligned}
$$

To avoid handling distributed delays (i.e. $S_{0}$ above), one makes use of (18):

$$
\mathcal{D} S_{0}=\frac{\tilde{\Delta}}{2 c},
$$

and apply $\mathcal{D}$ on both sides of (20) :

$$
\begin{aligned}
& \frac{G J c}{2} \mathcal{D}^{2} \tilde{\Delta} \Phi_{L}-\frac{1}{2} \mathcal{D} \Delta\left(I_{B} \ddot{\Phi}_{L}+T\left(\dot{\Phi}_{L}\right)\right) \\
& =\frac{\beta}{2} \mathcal{D} \Delta \dot{\Phi}_{L}-\frac{\beta}{G J}\left(\frac{\mathcal{D} \tilde{\Delta}-\zeta \tilde{\Delta}}{2 c}\right)\left(I_{B} \ddot{\Phi}_{L}+T\left(\dot{\Phi}_{L}\right)\right)-\mathcal{D} u_{T}
\end{aligned}
$$

which is a neutral system of order 3 with a single delay $\tau=c L$ that can be written as:

$$
\frac{G J c}{2} \alpha_{L} \ddot{\Phi}_{L}(t-2 \tau)-\frac{G J c}{2} \alpha_{L}^{-1} \ddot{\Phi}_{L}(t)+2 \frac{G J c}{2} \zeta \alpha_{L} \dot{\Phi}_{L}(t-
$$
$2 \tau)-2 \frac{G J c}{2} \zeta \alpha_{L}^{-1} \dot{\Phi}_{L}(t)+\zeta^{2} \frac{G J c}{2} \alpha_{L} \Phi_{L}(t-2 \tau)-$ $\zeta^{2} \frac{G J c}{2} \alpha_{L}^{-1} \Phi_{L}(t)-\frac{1}{2} I_{B} \alpha_{L} \dddot{\Phi}_{L}(t-2 \tau)-\frac{1}{2} I_{B} \alpha_{L}^{-1} \dddot{\Phi}_{L}(t)-$ $\frac{1}{2}\left(\alpha_{L} \ddot{\Phi}_{L}(t-2 \tau)+\alpha_{L}^{-1} \ddot{\Phi}_{L}(t)\right) T^{\prime}\left(\alpha_{L} \stackrel{\Phi}{L}_{L}(t-2 \tau)+\right.$ 
$\left.\alpha_{L}^{-1} \dot{\Phi}_{L}(t)\right)=\frac{\beta}{2} \alpha_{L} \ddot{\Phi}_{L}(t-2 \tau)+\frac{\beta}{2} \alpha_{L}^{-1} \ddot{\Phi}_{L}(t)+\frac{\beta \zeta}{2} \alpha_{L} \dot{\Phi}_{L}(t-$ $2 \tau)+\frac{\beta}{2} \alpha_{L}^{-1} \dot{\Phi}_{L}(t)-\frac{\beta I_{B} \alpha_{L}}{2 G J c} \dddot{\Phi}(t-2 \tau)+\frac{\beta I_{B} \alpha_{L}^{-1}}{2 G J} \dddot{\Phi}(t)-$ $\left(\alpha_{L} \ddot{\Phi}_{L}(t-2 \tau)-\alpha_{L}^{-1} \ddot{\Phi}_{L}(t)\right) T^{\prime}\left(\alpha_{L} \dot{\Phi}_{L}(t-2 \tau)-\alpha_{L}^{-1} \dot{\Phi}_{L}(t)\right)-$ $\dot{u}_{T}(t-\tau)-\zeta u_{T}(t-\tau)$.

which gives by collecting terms of the same order:

$$
\left\{\begin{array}{l}
\left(-\frac{\beta I_{B}}{\alpha_{L} 2 G J c}-\frac{I_{B}}{2 \alpha_{L}}\right) \dddot{\Phi}_{L}(t)+\left(\frac{\beta I_{B} \alpha_{L}}{2 G J c}-\frac{I_{B} \alpha_{L}}{2}\right) \dddot{\Phi}_{L}(t-2 \tau) \\
-\left(\frac{\beta}{2 \alpha_{L}}+\frac{G J c}{2 \alpha_{L}}\right) \ddot{\Phi}_{L}(t)+\left(\frac{G J c}{2} \alpha_{L}-\frac{\beta \alpha_{L}}{2}\right) \ddot{\Phi}_{L}(t-2 \tau) \\
-\left(2 \frac{G J c}{2 \alpha_{L}} \zeta+\frac{\beta}{2 \alpha_{L}}\right) \dot{\Phi}_{L}(t)+\left(2 \frac{G J c \zeta \alpha_{L}}{2}-\frac{\beta \zeta \alpha_{L}}{2}\right) \dot{\Phi}_{L}(t-2 \tau) \\
+\frac{\zeta^{2} G J c}{2 \alpha_{L}} \Phi_{L}(t)+\frac{\zeta^{2} G J c}{2 \alpha_{L}} \Phi_{L}(t-2 \tau) \\
=\frac{1}{2}\left(\alpha_{L} \ddot{\Phi}_{L}(t-2 \tau)+\alpha_{L}^{-1} \ddot{\Phi}_{L}(t)\right) T^{\prime}\left(\alpha_{L} \dot{\Phi}_{L}(t-2 \tau)+\alpha_{L}^{-1} \dot{\Phi}_{L}(t)\right) \\
-\left(\alpha_{L} \ddot{\Phi}_{L}(t-2 \tau)-\alpha_{L}^{-1} \ddot{\Phi}_{L}(t)\right) T^{\prime}\left(\alpha_{L} \dot{\Phi}_{L}(t-2 \tau)-\alpha_{L}^{-1} \dot{\Phi}_{L}(t)\right) \\
-\dot{u}_{T}(t-\tau)-\zeta u_{T}(t-\tau) .
\end{array}\right.
$$

\section{REFERENCES}

[1] Aarsnes, U.J.F., Aamo, O.M. (2016). Linear stability analysis of selfexcited vibrations in drilling using an infinite dimensional model. Journal of Sound and Vibration 360(6), 239-259.

[2] Mounier H., Greco L. (2016). Modelling and Structural Properties of Distributed Parameter Wind Power Systems. Proceeding of the 22nd International Symposium on Mathematical Theory of Networks and Systems.

[3] Knüppel, T., Woittennek, F., Boussaada, I., Mounier, H., Niculescu, S.I. (2013). Flatness-based control for a non-linear spatially distributed model of a drilling system. Low Complexity Controllers for Time Delay Systems, Springer series Advances in Delays and Dynamics.

[4] Boussaada, I., Mounier, H., Niculescu, S.I., Cela, A. (2012). Analysis of drilling vibrations: a time delay system approach. 20th Mediterranean Conference on Control and Automation MED, Barcelona, Spain.

[5] Woittennek, F., Mounier, H. (2010). Controllability of networks of spatially one-dimensional second order p.d.e. An algebraic approach. SIAM Journal on Control and Optimization, 48, pp.3882-3902.

[6] Depouhon, A., Detournay, E. (2014). Instability regimes and selfexcited vibrations in deep drilling systems. Journal of Sound and Vibration, 333(7), 2019-2039.

[7] J. K. Hale and S. M. Verduyn Lunel. Introduction to functional differential equations, volume 99 of Applied Mathematics Sciences. Springer Verlag, New York, 1993.

[8] Germay, C., Denoël, V., Detournay, E. (2009). Multiple mode analysis of the self-excited vibrations of rotary drilling systems. Journal of Sound and Vibration, 325(1-2), 362-381.

[9] I. Boussaada and S.-I. Niculescu, "Tracking the algebraic multiplicity of crossing imaginary roots for generic quasipolynomials: A Vandermonde-based approach," IEEE Transactions on Automatic Control, vol. 61, pp. 1601-1606, 2016.

[10] — "Characterizing the codimension of zero singularities for timedelay systems," Acta Applicandae Mathematicae, vol. 145, no. 1, pp. 47-88, 2016.

[11] _ _ "Computing the codimension of the singularity at the origin for delay systems: The missing link with Birkhoff incidence matrices," 21 st International Symposium on Mathematical Theory of Networks and Systems, pp. $1-8,2014$.

[12] I. Boussaada, H. Unal, and S.-I. Niculescu, "Multiplicity and stable varieties of time-delay systems: A missing link," in Proceeding of the 22nd International Symposium on Mathematical Theory of Networks and Systems, 2016, pp. 1-6.

[13] G. Pólya and G. Szegô, Problems and Theorems in Analysis, Vol. I: Series, Integral Calculus, Theory of Functions. New York, Heidelberg, and Berlin: Springer-Verlag, 1972.
[14] J. Vanbiervliet, K. Verheyden, W. Michiels, and S. Vandewalle, "A nonsmooth optimisation approach for the stabilisation of time-delay systems," ESAIM: COCV, vol. 14, no. 3, pp. 478-493, 2008.

[15] T. Vyhlidal and P. Zitek, "Mapping based algorithm for largescale computation of quasi-polynomial zeros," IEEE Transactions on Automatic Control, vol. 54, no. 1, pp. 171-177, 2009.

[16] T. Mori, N. Fukuma, and M. Kuwahara, "On an estimate of the decay rate for stable linear delay systems," International Journal of Control, vol. 36, no. 1, pp. 95-97, 1982.

[17] N. D. Hayes, "Roots of the transcendental equation associated with a certain difference-differential equation," Journal of the London Mathematical Society, vol. s1-25, no. 3, pp. 226-232, 1950.

[18] I. Boussaada, S. Tliba, S.-I. Niculescu, H. U. Unal, and T. Vyhlidal, "Further remarks on the effect of multiple spectral values on the dynamics of time-delay systems. application to the control of a mechanical system," Linear Algebra and its Applications, 2017.

[19] I. Boussaada, S.-I. Niculescu, S. Tliba, and T. Vyhlidal, "On the coalescence of spectral values and its effect on the stability of timedelay systems: Application to active vibration control," Procedia IUTAM, vol. 22, no. Supplement C, pp. 75 - 82, 2017.

[20] I. Boussaada, S.-I. Niculescu, and K. Trabelsi, "Towards a decay rate assignment based design for time-delay systems with multiple spectral values," in Proceeding of the 23nd International Symposium on Mathematical Theory of Networks and Systems, 2018, pp. 864871.

[21] I. Boussaada and S.-I. Niculescu, "On the dominancy of multiple spectral values for time-delay systems with applications," in To appear in: Proceeding of The 14th IFAC Workshop on Time Delay Systems, 2018, pp. 1-6.

[22] I. Boussaada, H. Mounier, S.-I. Niculescu, and A. Cela, "Analysis of drilling vibrations: A time-delay system approach," in Proceedings of The 20th Mediterranean Conference on Control and Automation, 2012, pp. 1-5.

[23] M. S. Marquez, I. Boussaada, H. Mounier, and S.-I. Niculescu, Analysis and Control of Oilwell Drilling Vibrations, ser. Advances in Industrial Control. Springer, 2015.

[24] Ghasemloonia, A., Rideout, D.G., Butt, S.D. (2015). A review of drillstring vibration modeling and suppression methods. Journal of Petroleum Science and Engineering, 131, 150-164.

[25] Henneuse, H. (1992). Surface detection of vibrations and drilling optimization: field experience. Proceedings of IADC/SPE Drilling Conference, New Orleans, Lousiana, 409-423.

[26] Michiels, W., Niculescu, S.I. (2007). Stability and stabilization of time-delay systems: an eigenvalue-based approach. Society for Industrial and Applied Mathematics (SIAM), Advances in Design and Control, Philadelphia, PA.

[27] Kamel, J.M., Yigit, A.S. (2014). Modeling and analysis of stick-slip and bit bounce in oil well drillstrings equipped with drag bits. Journal of Sound and Vibration, 333, 6885-6899.

[28] Khalil, H.K. (2002). Nonlinear systems, 3rd Edition, Prentice hall Upper Saddle River.

[29] Leine, R.I. (1997). Literature survey on torsional drillstring vibrations. Report number: WFW 97.069, Division of Computational and Experimental Mechanics, Department of Mechanical Engineering Eindhoven University of Technology, The Netherlands.

[30] Mason, J.S., Sprawls, B.M. (1998). Addressing BHA whirl: the culprit in mobile bay. SPE Drilling and Completion, 13(4), 231-236.

[31] Rasvan, V. (1975). A method for distributed parameter control systems and electrical networks analysis. Rev. Roumaine Sci. Techn. Serie Electrotechn. Energ. 20, 561-566.

[32] Saldivar, B., Knüppel, T., Woittennek, F., Boussaada, I., Mounier, H., Niculescu, S.I. (2014). Flatness-based control of torsional-axial coupled drilling vibrations. 19th World Congress of the International Federation of Automatic Control, Cape Town, South Africa.

[33] J. Zhou and M. Krstic, (2016). Predictor control for stabilizing pressure in a managed pressure drilling system under time delay, Journal of Process Control, vol. 40, pp. 106-118, 2016.

[34] Sagert, C., Di Meglio, F., Krstic, M., Rouchon, P. (2013). Backstepping and flatness approaches for stabilization of the stick-slip phenomenon for drilling. IFAC Symposium on System Structure and Control, Grenoble, France.

[35] Saldivar, M.B., Mondié,S.-I. Niculescu, H. Mounier, I. Boussaada. (2016). A control oriented guided tour in oilwell drilling vibration modeling, Annual reviews in Control,, 42, pp. 100-113. 